\section{RE: Appendiceal} Immunoglobulin G4-Related Disease Mimicking Appendiceal Tumor or Appendicitis: A Case Report

\section{Dong Hyang Kwon, MD,}

Bhaskar Venkata Kallakury, MD

All authors: Departments of Pathology and Laboratory Medicine, MedStar Georgetown University Hospital, Washington, DC 20007, USA

Keywords: IgG4-related disease, Appendix, CT

We read with great interest the article by Kim et al. (1), titled "Appendiceal immunoglobulin G4-related disease mimicking appendiceal tumor or appendicitis: a case report," that described the first report of immunoglobulin G4 (IgG4)-related disease involving appendix. The authors emphasized the significance of recognizing this relatively recent entity in the differential diagnosis for a massforming lesion to avoid unnecessary surgery.

We encountered a similar scenario, in which a patient was presented with signs and symptoms of appendicitis, accompanied by a mass-forming lesion. This raised a clinical concern of neoplasm, therefore hemicolectomy was conducted as a curative option. Upon pathological evaluation, the lesion revealed a chronic fibro-inflammatory process with lymphoplasmacytic infiltrate of which, the IgG 4 subset accounted for $>50$ percent overall and $>100$ / high power field in high density areas accompanied by

Received June 27, 2017; accepted after revision June 28, 2017. Corresponding author: Dong Hyang Kwon, MD, Department of Pathology and Laboratory Medicine, MedStar Georgetown University Hospital, 3900 Reservoir Road NW, Washington, DC 20007, USA.

- Tel: (1202) 687-8935 - Fax: (1202) 784-3635

- E-mail: donghyang.kwon@gunet.georgetown.edu This is an Open Access article distributed under the terms of the Creative Commons Attribution Non-Commercial License (http://creativecommons.org/licenses/by-nc/4.0) which permits unrestricted non-commercial use, distribution, and reproduction in any medium, provided the original work is properly cited. obliterative phlebitis and arteritis, confirming diagnosis as IgG4-related appendiceal disease. We agree with the authors that IgG4-related disease must be better recognized to avoid unnecessary or excessive surgery. However, this diagnosis may only be established using stringent histologic criteria. We share our experience to increase diagnostic awareness of IgG4-related disease and add comments on diagnostic criteria.

An international committee comprised of 35 experts formulated guidelines for pathological diagnosis at the International Symposium on IgG4-related disease held in Boston, Massachusetts in 2012 (2). Histopathological features included 1) a dense lymphoplasmacytic infiltrate, 2) a storiform pattern of fibrosis, and 3) obliterative phlebitis. The committee concluded that IgG4 ratio is of secondary significance because other inflammatory lesions may be present with a high number of IgG4+ plasma cells because of an abundance of plasma cells. Other minor features included eosinophilia, phlebitis without obliteration, increased IgG4 in serum level, and response to glucocorticoid treatment.

Kim et al. (1) described subserosal inflammatory cell infiltration mostly constituted by plasma cells with immunohistochemistry stains validating significantly increased IgG4 subset. The authors stated the absence of more specific and diagnostic storiform fibrosis or obliterative phlebitis. In contrast, our case (manuscript under preparation), demonstrated all three histopathologic features, making it the first IgG4-related disease of appendicitis to our knowledge that fully satisfied all the pathological diagnostic criteria for this disease. Our case was also unique in that there was a concomitant significant dendritic cell hyperplasia marked by diffuse positivity for S100, that may play a pathophysiologic role in this entity and by itself may mimic a mass-forming lesion.

In conclusion, IgG4-related disease may be present in rare locations, including the appendix. This newly described entity of the last decade deserves increased recognition due to clinical implication and morbidity. 


\section{REFERENCES}

1. Kim HS, Kang WK, Chung DJ. Appendiceal immunoglobulin G4related disease mimicking appendiceal tumor or appendicitis: a case report. Korean J Radiol 2016;17:56-58

2. Deshpande V, Zen Y, Chan JK, Yi EE, Sato Y, Yoshino T, et al. Consensus statement on the pathology of IgG4-related disease. Mod Pathol 2012;25:1181-1192 